\title{
THEORY ON PLANE CURVES IN NON-METRICAL ANALYSIS SITUS*
}

BY

\section{OSWALD VEBLEN}

\section{$\S 1$. Introduction.}

JORDAN's $\dagger$ explicit formulation of the fundamental theorem that a simple closed curve lying wholly in a plane decomposes the plane into an inside and an outside region is justly regarded as a most important step in the direction of a perfectly rigorous mathematics. This may be confidently asserted whether we believe that perfect rigor is attainable or not. His proof, however, is unsatisfactory to many mathematicians. It assumes the theorem without proof in the important special case of a simple polygon $\ddagger$ and of the argument from that point on, one must admit at least that all details are not given.

The work of Schoenflies, $\S$ especially in formulating a converse theorem has thrown much light on its relation to the theory of point sets and Analysis Situs in general, and elegant proofs under restrictive hypotheses have been given by Ames $\|$ and Buiss.** All these discussions make more or less use of the ideas of analysis, thus implying either an axiom to the effect that a plane is a doubly extended number-manifold or a set of congruence axioms. Either of these hypotheses imposes a restriction upon the formal generality of Analysis Situs as a science independent of the magnitude of the figures treated.

* Presented to the Society at the St. Louis meeting, September 17, 1904, under the title, The fundamental theorem of Analysis Situs. Received for publication August 22, 1904.

†C. Jordan, Cours d'Analyse, Paris, 1893, 2d ed., p. 92.

$\ddagger$ This case was under discussion at the University of Chicago in 1901-02 in connection with Professor MOoRE's seminar on Foundations of Geometry. Mr. N. J. LeNNES gave a proof in his master's thesis (1903), Theorems on the simple polygon and polyhedron. Another proof appears as theorem 28 in the writer's dissertation (for reference, see footnote below). The present paper owes much to the discussions of the subject that have taken place under the leadership of Professor MOORE.

§. Schomplite, Ueber einen grundlegenden Satz der Analysis Situs, Nachrichten der Göttinger Gesellschaft der Wissenschaften, 1902, p. 185; Beiträge zur Theorie der Punktmengen, Mathematische Annalen, Vol. 58 (1903), p. 195.

$\|$ L. D. AMES, On the theorem of Analysis Situs relating to the division of the plane or of space by a closed curve or surface, Bulletin of the American Mathematical Society (2), vol. 10 (1904), p. 301.

${ }^{* *}$ G. A. BuIss, The exterior and interior of a plane curve, Bulletin of the American Mathematical Society (2), vol. 10 (1904), p. 398. 
In the following pages an attempt is made to discuss the theorem of Jordan and a number of related questions under considerably more general hypotheses than are employed in any of the works referred to above. This undertaking practically results in a stacement in logical terms of a body of information that formerly was used without explicit formulation and recently has been cramped by unnecessary restrictions. In other words we are inquiring how wide may be the application of our intuitive notion of a plane curve.*

The arguments and definitions are based upon axioms I-VIII, XI of the system adopted in the writer's dissertation $\dagger$ to which refer all the citations not otherwise indicated. These axioms are sufficient to determine the intersectional properties of straight lines, the ordinal relations $\ddagger$ of points on a straight line, and continuity. We accordingly assume nothing about analytic geometry, the parallel axiom, congruence relations, nor the existence of points outside a plane. For example, the theory is as valid in the non-desarguesian geometries of HiLBERT and Moulton $\S$ as in the geometries of EucLid and Lobatchewsky, and is of course as applicable in pure analysis as in geometry. $\|$

The reader who prefers the JoRDAN definition of a simple curve (which according to $\S 4$ is equivalent to ours for purposes of analysis) and does not care about the question of non-metrical hypotheses, may conveniently begin with $\S 5$. A relatively simple proof of the theorem of JORDAN about the decomposition of the plane which applies to any simple closed curve having a straight line inter-

* The general problem of the "mathematics of precision" may be stated in similar terms.

$\dagger$ O. Veblen, $A$ System of Axioms for Geometry, Transactions of the A merican Mathematical Society, vol. 5 (1904), pp. 343-384.

$\ddagger$ The line is open, i. e., between every two points there is a third, and the order $A B C$ excludes $B A C$ and $A C B$. Single elliptic geometry and projective geometry are therefore excluded unless a properly chosen cut is introduced.

$\S$ F. R. Moulton, A Simple non-desarguesian Geometry, these Transactions, vol. 3 (1902), p. 192.

$\|$ As to the applicability of our results in analysis, it seems desirable to add a remark which, though obvious from the point of view of "foundations of mathematics," may be of service to some readers who are not directly interested in this point of view. Numerical analysis is ordinarily thought of as founded on the concept of the positive integers. In terms of these a proof of existence can be given of a set of elements, or quantities, satisfying the postulates of the system of rational numbers, positive and negative. In terms of the rational numbers, in turn, can be given a proof of the existence of elements satisfying the postulates of the continuous real number system. Finally, the processes of analysis have to do with pairs of real numbers $(x, y)$. The set of all such number-pairs is a set of objects about which (with proper definition of the term "order") our axions I-VIII, XI are true theorems. From the axioms of analysis, the line of deduction of our theorems is therefore clear and simple. Not only that, but we may add that any theorem or any definition rigorously based on the assumptions of geometry is ipso facto a theorem or definition of analysis. Such considerations as these justify the assertion that while much may be lost in elegance and simplicity, nothing is gained in rigor by the banishment of geometrical language and geometrical styles of exact reasoning from pure analysis. (Of course, under sufficiently strong geometrical axioms, these remarks may be reversed and applied to the rôle of analysis in geometry.) 
val is to be obtained by considering theorem 8 applied to a triangular region, theorem 9 applied to a simple polygon, corollary 2 of theorem 9 , lemma $B$, theorem 10 and lemma $C$. This " reduced proof" could in turn be slightly modified so as to apply to any curve having at least one non-cuspidal tangent.

\section{§ 2. Non-metrical definition of limit point.}

For the definition of the terms, triangle, polygon, broken line, triangular region, separate, decompose, the reader is referred to $\S 4$, chapter II. Of the theorems there proved we assume for the present purpose only that a triangle decomposes a plane in which it lies into two regions, an interior and an exterior.

Definirion 1. A triangular region is the interior of a triangle. A geometrical limit point of a set of points, $[X],{ }^{*}$ in a plane is a point $P$ such that every triangular region including $P$ includes a point $X$, distinct from $P$. A triangular region including a point is called a neighborhood of the point.

The continuity axiom was assumed for only one segment of a straight line and proved by projection for all lines. In like manner by projection it can be proved that for every point, $P$, of any line there exists a numerably infinite set of segments $\left[\sigma_{\nu}\right](\nu=1,2, \cdots)$ such that $\sigma_{\nu}$ contains $\sigma_{\nu+1}$ and such that $P$ is the only point that lies on every $\sigma_{v}$. It is an easy consequence of this that for every point in a plane there exists a set of triangular regions $\left[t_{\nu}\right]$ with a similar property. We also prove without difficulty the theorem that a limit point of a set of limit points of a set of points, $\left[X_{*}\right]$, is itself a limit point of $[X]$.

Definition 2. A region is a set of points, any two of which are points of at least one broken line composed entirely of points of the set. An interior point of a region, $R$, is one that can be surrounded by a triangle containing only points of $R$. Consequently, an interior point of $R$ is a geometrical limit point of no set of points that does not contain points of $R$. A frontier point of a region $R$ is a point or geometrical limit point of $R$ not an interior point, i. e., it is a limit point both of $R$ points and of not $R$ points. An exterior point of $R$ or a point exterior to $R$ is any point neither an interior nor a frontier point of $R$. The frontier or boundary of a region is a set of all frontier points. An open region contains no frontier points. A closed region contains all its frontier points.

One of the most familiar examples of an open region is obtained by letting $[C]$ stand for a closed set of points and $[P]$ for the set of all points that can be joined with a point $P_{0}$ not of $[C]$ by broken lines not meeting $[C] ;[P]$ is an open region.- It is to be noted that the points exterior to a region $[P]$, if such exist, need not constitute only a single region.

* The notation $[X]$ denotes a set of elements any one of which is denoted by $X$ alone or with suffixes. If we wish to indicate that the set is ordered we use $\{X\}$ instead of $[X]$. 


\section{§3. Definition of simple curve.}

Simple curves, closed and unclosed, are composed of sets of points subject to certain conditions which we arrange in the following groups :

$A$. Linear order. Among the points of a set of points $\{\mathbf{P}\}$ there exists a relation, $\otimes$, which we may read precedes, such that:

1. $\{\mathbf{P}\}$ contains at least two points.

2. If $\mathrm{P}_{1}$ and $\mathrm{P}_{2}$ are any two distinct points of $\{\mathrm{P}\}$, then either $\mathrm{P}_{1} \otimes \mathrm{P}_{2}$ or $\mathbf{P}_{2} \otimes \mathbf{P}_{1}$.

3.* If $\mathrm{P}_{1} \otimes \mathrm{P}_{2}$, then not $\mathrm{P}_{2} \otimes \mathrm{P}_{1}$.

4. If $\mathrm{P}_{1} \otimes \mathrm{P}_{2}$ and $\mathrm{P}_{2} \otimes \mathrm{P}_{3}$, then $\mathrm{P}_{1} \otimes \mathrm{P}_{3}$.

$B$. Ordinal continuity.

1. If $\mathrm{P}_{1}$ and $\mathrm{P}_{3}$ are any two points of $\{\mathbf{P}\}$, such that $\mathrm{P}_{1} \otimes \mathrm{P}_{3}$, then there is a point $\mathbf{P}_{2}$ of $\{\mathbf{P}\}$ such that $\mathbf{P}_{1} \otimes \mathbf{P}_{2}$ and $\mathbf{P}_{2} \otimes \mathbf{P}_{3}$.

2. If every point of $\{\mathrm{P}\}$ belongs to $\left[\mathrm{P}_{1}\right]$ or $\left[\mathrm{P}_{2}\right]$, two infinite subsets of $\{\mathbf{P}\}$ such that for every $\mathbf{P}_{1}$ and $\mathbf{P}_{2}, \mathbf{P}_{1} \otimes \mathbf{P}_{2}$, then there is a point $\mathbf{P}^{\prime}$ such that for every $\mathrm{P}_{1}$ and $\mathrm{P}_{2}$ distinct from $\mathrm{P}^{\prime}, \mathrm{P}_{1} \otimes \mathrm{P}^{\prime}$ and $\mathrm{P}^{\prime} \otimes \mathrm{P}_{2}$.

C. Geometrical Continuity.

1. Let $\mathrm{P}_{0}$ be any point of $\{\mathbf{P}\}$ for which there is an infinity of points $\mathbf{P}^{\prime}$ such that $\mathbf{P}^{\prime} \otimes \mathrm{P}_{0}$. Denote the set of all such points by $\left[\mathrm{P}^{\prime}\right]$; then for every triangular region, $t$, including $\mathrm{P}_{0}$, there is a point of $\left[\mathrm{P}^{\prime}\right], \mathrm{P}_{t}^{\prime}$ such that $t$ includes all points of $\left[\mathrm{P}^{\prime}\right]$ for which $\mathrm{P}_{t}^{\prime} \otimes \mathrm{P}^{\prime}$.

2. Let $\mathrm{P}_{0}$ be any point of $\{\mathbf{P}\}$ for which there is an infinity of points $\mathrm{P}^{\prime \prime}$ such that $\mathrm{P}_{0} \otimes \mathrm{P}^{\prime \prime}$. Denote the set of all such points by $\left[\mathrm{P}^{\prime \prime}\right]$; then for every triangular region, $t$, including $\mathrm{P}_{0}$ there is a point of $\left[\mathrm{P}^{\prime \prime}\right], \mathrm{P}_{t}^{\prime \prime}$ such that $t$ includes all points of $\left[\mathrm{P}^{\prime \prime}\right]$ for which $\mathrm{P}^{\prime \prime} \otimes \mathrm{P}_{t}^{\prime \prime}$.

Definition 3. By the term arc or arc of curve is meant a set of points $\{\mathrm{P}\}$ satisfying conditions $A, B, C$ and including two points $\mathrm{P}_{1}, \mathrm{P}_{2}$ such that every point $\mathrm{P}$, distinct from $\mathrm{P}_{1}$ and $\mathrm{P}_{2}$, satisfies the further conditions that $P_{1} \otimes P$ and $P \otimes P_{2}$. The are is said to join $P_{1}$ and $P_{2}$ which are called its end-points.

Definition 4. A simple closed curve, $j$, is a set of points, $\{J\}$, consisting of two arcs joining two points $J_{1}$ and $J_{2}$ but having in common no points other than $J_{1}, J_{2}$.

Theorem 1. Any two points of $j$ may be taken as the points, $J_{1}, J_{2}$ in the above definition.

The proof of this theorem is here omitted as it involves no difficulty. The existence of sets of points satisfying the conditions of our definitionis proved by the examples of an interval of a straight line, which is an arc, and the boundary of a simple polygon, which is a simple closed curve. We shall use the letter $j$,

\footnotetext{
* Frem this it follows that if $P_{1} \otimes P_{2}$, then $P_{1} \neq P_{2}$.
} 
to denote a simple closed curve, in honor of Camille Jordan. The term arc of course does not cover the most general case of an unclosed curve. On the other hand the conditions $A, B, C$ are too general to define an unclosed curve since they are satisfied, for example, by the boundary of a triangle exclusive of one vertex. We therefore set down the following condition which is evidently satisfied by an arc.

Definition 5. A simple unclosed curve is a set of points $\{C\}=\mathrm{c}$ that satisfies conditions $A, B, C$ and also the following:

D. If $C$ is any point of the curve, no point except $C$ is a limit point (in the geometrical sense of definition 1) both of the set of all points $C^{\prime}$ such that $C^{\prime} \otimes C$ and of the set of all points $C^{\prime \prime}$ such that $C \otimes C^{\prime \prime}$.

Any simple closed or simple unclosed curve is called a simple curve. For a set of points satisfying conditions $A, B$ and $C$, it is evident that there hold all the propositions usually proved in the theory of linear point-sets with the exception of those that involve the length of intervals. We may mention particularly the propositions of section 5, chapter II, including the HeINE-BorEL theorem and the definition of ordinal limit point, the properties of point-free intervals in connection with closed sets, and the proposition that a line cannot be separated into two subsets each of which includes all its limit points. We do not stop here to prove these propositions though we make use of the last one in the following theorem.

Definition 6. A relation satisfying conditions $A, B, C$ is called a sense. A sense in which $\mathrm{P}_{1} \otimes \mathrm{P}_{2}$ is said to be from $\mathrm{P}_{1}$ to $\mathrm{P}_{2}$.

Theorem 2. From one point to another upon a simple unclosed curve there is one and but one sense, while upon a simple closed curve there are two and but two senses.

Proof. We have to show that if $\rho$ is any relation satisfying the conditions $A, B, C$ and $D$ imposed on $\otimes$, then if $\mathrm{P}_{1} \rho \mathrm{P}_{2}$ implies for one pair $\mathrm{P}_{1} \mathrm{P}_{2}$ of $\{\mathrm{P}\}$ that $\mathrm{P}_{1} \otimes \mathrm{P}_{2}, \mathrm{P}_{1} \rho \mathrm{P}_{2}$ implies that $\mathrm{P}_{1} \otimes \mathrm{P}_{2}$ for every pair $\mathrm{P}_{1} \rho \mathrm{P}_{2}$ of $\{\mathrm{P}\}$.

If $\mathrm{P}_{1}$ is any point of $\{\mathrm{P}\}$, let $\left[\mathrm{P}^{\prime}\right]$ be the set of all points such that simultaneously $\mathrm{P}_{1} \rho \mathrm{P}^{\prime}$ and $\mathrm{P}_{1} \otimes \mathrm{P}^{\prime}$. Every limit point $\overline{\mathrm{P}}\left(\overline{\mathrm{P}} \neq \mathrm{P}_{1}\right)$ of $\left[\mathrm{P}^{\prime}\right]$ with respect to the sense $\rho$ must by conditions $A, B$ and $C$ be such that $\mathrm{P}_{1} \rho \mathrm{P}^{\prime}$. Moreover $\mathrm{P}$, by condition $C$, is a geometrical limit point of $\left[\mathrm{P}^{\prime}\right]$. But in view of condition $D, \overline{\mathrm{P}}$ being a point of $\{\mathrm{P}\}$ and a geometrical limit point of points $\mathrm{P}^{\prime}$ such that $\mathrm{P}_{1} \otimes \mathrm{P}^{\prime}$ must be such that $\mathrm{P}_{1} \otimes \mathrm{P}$; otherwise $\overline{\mathrm{P}}\left(\mathrm{P} \neq \mathrm{P}_{1}\right)$ would be a geometrical limit point both of points $\overline{\mathrm{P}}$ such that $\overline{\mathrm{P}} \otimes \mathrm{P}_{1}$ and of points $\mathrm{P}^{\prime}$ such that $\mathrm{P}_{1} \otimes \mathrm{P}^{\prime}$.

Therefore the set $\left[\mathrm{P}^{\prime}\right]$, if existent, contains all its limit points with respect to the sense $\rho$, except the point $\mathrm{P}_{1}$. Similarly the set of all points $\mathrm{P}^{\prime \prime}$, such that simultaneously $\mathrm{P}_{1} \rho \mathrm{P}^{\prime \prime}$ and $\mathrm{P}^{\prime \prime} \otimes \mathrm{P}_{1}$ must, if existent, contain all its limit points with respect to the sense $\rho$. Therefore, since the set of points $\left\{P_{2}\right\}$ 
such that $\mathrm{P}_{1}<\mathrm{P}_{2}$ cannot consist of two subsets, each closed with the exception of $P_{1}$, every point $P_{2}$ must either be such that $P_{1} \otimes P_{2}$ or every point $P_{2}$ must be such that $P_{2} \otimes P_{1}$. From this result the conclusion of our theorem follows at once.

Definition 7. If with respect to any sense on a curve, $\mathrm{P}_{1} \otimes \mathrm{P}_{2}$ and $\mathrm{P}_{2} \otimes \mathrm{P}_{3}$, $P_{2}$ is between $P_{1}$ and $P_{3}$ in that sense. The set of all points between $P_{1}$ and $P_{3}$ in the given sense is called a segment $\mathrm{P}_{1} \mathrm{P}_{2} \mathrm{P}_{3}$ whose end-points are $\mathrm{P}_{1}, \mathrm{P}_{3}$. The segment and its end-points together constitute an arc or interval of the curve. On a simple unclosed curve, if $\mathrm{P}_{1} \otimes \mathrm{P}_{2} \otimes \mathrm{P}_{3}, \mathrm{P}_{2}$ is said to separate $\mathrm{P}_{1}$ and $\mathrm{P}_{3}$. On any simple curve if $\mathrm{P}_{1} \otimes \mathrm{P}_{2} \otimes \mathrm{P}_{3} \otimes \mathrm{P}_{4}, \mathrm{P}_{1}$ and $\mathrm{P}_{3}$ are said to separate and be separated by $\mathrm{P}_{2}$ and $\mathrm{P}_{4}$. If a set $\left[\mathrm{P}_{\nu}\right](\nu=1,2,3, \ldots)$ is such that $\mathrm{P}_{\nu} \otimes \mathrm{P}_{\nu+1}$, the points $\mathrm{P}_{\nu}$ are said to be in the order along the curve, $\mathrm{P}_{1} \mathrm{P}_{2} \mathrm{P}_{3} \ldots \mathrm{P}_{\nu} \mathrm{P}_{\nu+1} \cdots$. A point $\mathrm{P}_{0}$ is the first of a set $[\mathrm{P}]$ if $\mathrm{P}_{0} \otimes \mathrm{P}$ for every $\mathrm{P} \neq \mathrm{P}_{0} ; \mathrm{P}_{1}$ is the last of the set $[\mathrm{P}]$ if $\mathrm{P} \otimes \mathrm{P}_{1}$ for every $\mathrm{P} \neq \mathrm{P}_{1}$.

Either of the relations of "betweenness" or "separation" which are here defined in terms of "precedence" could have been used as fundamental* and a definition of a simple curve equivalent to the above would have resulted. The deduction of the properties of these relations will be omitted.

\section{§4. Remarks on the definition of a simple closed curve.}

While the definitions of the preceding section are stated so as to apply only to plane curves, it is obvious that if one replaces triangles by tetrahedrons or the corresponding figures in space of more dimensions, the conditions $A, B, C$, etc., give a definition of a simple curve in space of any number of dimensions.

It may be of interest to note that when one passes from the realm of plane geometry, the distinction between metric and non-metric theory loses much of its importance. For if we add to our assumptions (axioms I-VIII, XI) the assumption (axiom IX) that there exists a point outside a plane, then it is possible to define the ideal elements of projective geometry (cf. chapter III) and by choosing among these ideal elements an "absolute" plane $\dagger$ and polar system to establish a projective theory of congruence. We are thus enabled to operate in the most general case by ordinary analytic geometry as if dealing with the whole or a limited region of euclidean space.

As to the relation of the above definition to the current definition $\ddagger$ in terms of a numerical parameter, it has not yet been determined whether, in the presence of axioms I-VIII, XI alone, the two definitions are or are not equivalent.

* Cf. B. Russeld, The Principles of Mathematics, Cambridge, 1903, chapters 24, 25. On the definition by postulate of "separation," see G. VAILATI, Sulle relazioni cii posizione tra punti d'una linea chiusa, Rivista di Matematica, vol. 5 (1895), p. 75 ; and also ibid., p. 183.

$\dagger$ That there always is an ideal plane depends in particular on axioms III and XI which determine that straight lines shall be open.

$\Varangle$ See JoRDAN, loc. cit. p. 90. 
If however we introduce axiom IX as indicated above or bring in a set of congruence axioms like HiLberT's group IV, then the two definitions can be shown to be equivalent by reference to a theorem of CANTOR.*

Let $a$ be any arc of a simple closed curve $j$. The points of $a$, excluding the end points, evidently constitute what CanTor calls a perfect set, i. e., with respect to the sense $\otimes$. Let $\left\{t_{n}\right\}$ denote a set of equilateral triangles concentric and similarly placed such that the lengths of the side of $t_{n}$ is $1 / n$. For every point $J$ of $a$ there is such a set of triangles $\left\{t_{n}\right\}_{J}$, having $J$ as a common center. Each triangle $t_{n}$ determines an arc $i_{n}$ of $j$ which lies wholly within $t_{n}$ (cf. condition $C$ ) and includes the central point $J$ of $t_{n}$. Among the arcs $i_{n}$, there is by the HeIne-Borel theorem applied to $a$, a finite subset such that every point of $a$ is interior to one of the arcs $i_{n}$. The end points of these ares that lie on $a$, excluding the end points of $a$, we denote by

$$
A_{n}^{\prime}, A_{n}^{2}, \ldots, A_{n}^{m_{n}} .
$$

The set of points $\left\{A_{n}^{k}\right\}$ is evidently numerable, is ordered according to one of the senses of $a$, and moreover is everywhere dense on $a$. For if it were not everywhere dense on $a$ there would be some interval $i$ of $a$ which for every $n$ lies wholly within some $i_{n}$ and therefore within some $t_{n}$; whereas two of its points are a certain distance apart greater than $1 / n$ for $n$ sufficiently great.

Now by the theorem of CANTOR cited above, any perfect set which possesses a numerable subset everywhere dense can be set in one-to-one reciprocal continuous correspondence, with the real numbers between 0 and 1 . Thus we have a continuous one-to-one correspondence of the points of any arc, and hence of any simple closed curve, with a numerical parameter, $t$. If a system of coördinates $(x, y)$ has been introduced, the simple closed curve may be expressed in parameter form by defining $x(t)$ as the abscissa of the point of $j$ that corresponds to $t$ and $y(t)$ as the ordinate of the same point. The continuity of $x(t)$ and $y(t)$ is evident.

Regarding the conditions of definition 4 as a set of postulates for the determination of the notion, simple curve, the proposition just proved is in effect that in the presence of axioms I-VIII, XI, together with IX or a set of congruence axioms, the system of postulates is "categorical." $\dagger$ The conditions are also independent; i. e., each item of the definition is indispensable to the full definition. To prove this we give a list of point-sets each of which satisfies all the conditions except one. Our independence proofs apply to conditions $A, B, C$, $D$ since the closed simple curve is defined in terms of the unclosed arc.

$A_{1} \cdot\{\mathrm{P}\}$ consists of one point.

${ }^{*}$ G. Cantor Zur Begrïndung der transfiniten Mengenleltre I, Mathe matische Annalen, vol. 46 (1895), p. 510.

$\dagger$ See vol. 5, p. 346, of these Transactions. 
$A_{2} \cdot\{\mathrm{P}\}$ consists of a straight segment $\mathrm{P}_{1} \mathrm{P}_{2}$ and a point $\mathrm{P}_{3}$ not on the straight line $\mathrm{P}_{1} \mathrm{P}_{2}$, the relation $\otimes$ referring to a fixed sense on the straight line $\mathrm{P}_{1} \mathrm{P}_{2}$.

$A_{3} .\{\mathrm{P}\}$ consists of two points $\mathrm{P}_{1}, \mathrm{P}_{2}$ with the conventions $\mathrm{P}_{1} \otimes \mathrm{P}_{2}, \mathrm{P}_{1} \otimes \mathrm{P}_{1}$, $\mathrm{P}_{2} \otimes \mathrm{P}_{2}$.

$A_{4} \cdot\{\mathrm{P}\}$ consists of seven points $\mathrm{P}_{1} \ldots \mathrm{P}_{7}$, the relation $\otimes$ being defined by the following table

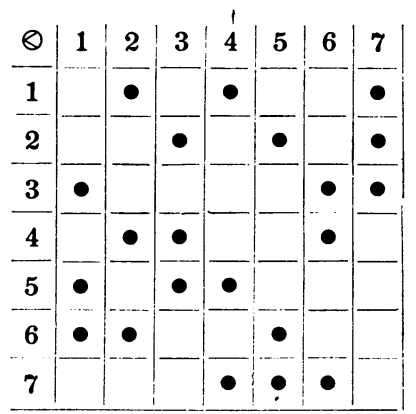

$B_{1}$. $\{\mathbf{P}\}$ consists of two points $\mathbf{P}_{1}$ and $\mathbf{P}_{2}$ with the convention $\mathbf{P}_{1} \otimes \mathbf{P}_{2}$.

$B_{2}:\{\mathbf{P}\}$ consists of all the points of a straight line with one exception, e being one of the two senses along the line.

$C_{1} \cdot\{\mathrm{P}\}$ consists of all the points of a straight line $\mathrm{P}_{1} \mathrm{P}_{2}$ with the exception of the point $P_{1}$ and the segment $P_{1} P_{2}$, $\odot$ being the sense from $P_{1}$ to $P_{2}$.

$C_{2} .\{\mathrm{P}\}$ consists of all the points of a straight line $\mathrm{P}_{1} \mathrm{P}_{2}$ with the exception of the point $P_{2}$ and the segment $P_{1} P_{2}$, \& being the sense from $P_{1}$ to $P_{2}$.

$D$. $\mathrm{P}$ consists of the points of a broken line $\mathrm{P}_{1} \mathrm{P}_{2} \mathrm{P}_{3} \mathrm{P}_{4}$, where $\mathrm{P}_{4}$ is a point of the segment $P_{1} P_{2}$, Q being the sense $P_{1} P_{2}$, and $P_{4}$ being counted as a point of $\mathrm{P}_{1} \mathrm{P}_{2}:$ This case shows the necessity of condition $\mathrm{D}$ in theorem 2 since $\theta$ may also be the sense along the broken line $\mathrm{P}_{1} \mathrm{P}_{4} \mathrm{P}_{3} \mathrm{P}_{2}$.

\section{§5. A simple curve as. a planar point set.}

Definition 8. A geometrically closed set of points is a set that includes all its geometrical limit points.

Theorem 3. If $[\mathrm{P}]$ is any geometrically closed set of points and $a$ any arc that does not have any point in common with $[\mathrm{P}]$, then (1) there exists a finite set of triangles $\left\{t_{n}\right\}$ such that every point of $a$ is interior to at least one $t_{n}$ and every point of $[\mathrm{P}]$ is exterior to every $t_{n}$, and (2) the two end points $A_{1} A_{2}$ of a can be joined by a broken line not meeting [P].

Proof. (1) If $A$ is any point of $a$ there must be a triangle, $t$, including $A$ and not including any point of $[\mathrm{P}]$; otherwise $A$ would be a limit point of [P]. By condition $c$, each of these triangles, $t$, determines an arc, $i$, of $a$ which lies entirely within $t$ and includes the point $A$ to which $t$ belongs. By 
the HeIne-Borel theorem applied to the arc $a$, there is a finite subset $\left[i_{n}\right]$ of the $\operatorname{arcs} i$ such that every point of $a$ belongs to one are $i_{n}$. The finite set of triangles, $t_{n}$, that determined these arcs $i_{n}$ is the set required by conclusion (1) of the theorem.

(2) The end points of the ares $i_{n}$ constitute a finite set of points which we take as ordered by the sense of $a$ from $A_{1}$ to $A_{2}$. The broken line* joining these points taken in order is such that each side lies within a triangle $t$ and therefore cannot meet $[\mathrm{P}]$.

Corollary. If $[\mathrm{P}]$ is any geometrically closed set of points and $Q_{0}$ a point not of $[\mathrm{P}]$, then $Q_{0}$ and the set of points, $Q$, that can be joined to $Q_{0}$ by arcs not meeting $[\mathrm{P}]$ constitute an open region.

The following theorem is a direct consequence of definitions 4 and 5 and its proof as well as that of theorem 5 is omitted.

Theorem 4. About any point of a segment of a simple curve there is a triangle which includes no points of the curve not on the segment.

In the sense of definition 8 , a straight line is a geometrically closed set. A straight line, however, lacks a property possessed by any one of its intervals, namely that every infinite subset has a limit point. For this kind of set we introduce the phrase "finitely closed" because any such set can be enclosed by a finite set of triangles. This property, however, is not used and not proved in the present paper.

Definition 9. A finitely closed set of points is a geometrically closed set of which every infinite subset possesses a geometrical limit point. A finitely closed set, every point of which is a geometrical limit point, is a finitely perfect set. A finitely perfect set of points which cannot consist entirely of two closed subsets is called a coherent set of points. $\dagger$

Theorem 5. A closed curve or an arc of curve is a finitely perfect set of points which cannot consist entirely of two subsets, each of which includes all its limit points. In other words a closed curve or an arc of curve is a coherent set of points.

THEOREM 6. If every point of a coherent set of points $[A]$ is on a simple curve $c$, closed or unclosed, then $[A]$ is an interval of $c$.

Proof. If $[A]$ were not an arc of $c$ there must in case $c$ is unclosed be one, and in case $c$ is closed, two points, $C_{1}, C_{2}$, of $C$ not on $a$ which separate the points of $c$ into two sets, $c^{\prime}, c^{\prime \prime}$ each containing points of $[A]$. Let $\left[A^{\prime}\right]$ denote the points common to $[A]$ and $c^{\prime}$ and $\left[A^{\prime \prime}\right]$ denote the points common to $[A]$ and $c^{\prime \prime}$. Every geometrical limit point of $\left[A^{\prime}\right]$ would be a

\footnotetext{
* This broken line of course need not be simple. A broken line with multiple points has a sense independent of the definition of sense on a simple curve. See chapter II, $\$ 4$.

†This is the "Begriff des Zusammenhangs" of JoRdAN and Schoenflies. Cf. SchoknFLIES, Mathematische Annalen, vol. 58 (1903), p. 208.
} 
geometrical limit point of $[A]$, therefore a point of $[A]$, and hence a point of $c$. Being a point of $c$ and a geometrical limit point of $c^{\prime}$, by theorem 4 , it would be a point of $c^{\prime}$ and hence a point of $\left[A^{\prime}\right] . \quad\left[A^{\prime}\right]$ would therefore be a closed set and by parity of reasoning $\left[A^{\prime \prime}\right]$ also would be closed and thus the definition of $[A]$ would be contradicted.

Corollary. If every point of an arc, $a$, is on a simple curve, $c$, then $a$ is an interval of $c$.

Theonem 7. If $c$ is any simple curve, any triangle, $t$, of the plane includes points not on $c$.

Proof. Let $a$ be a straight line interval lying wholly within $t$. By theorem 6, $a$ either contains points not on $c$, in which case our conclusion holds, or $a$ is an arc of $c$. In the latter case, by theorem 4, a triangle, $t^{\prime}$, exists about any interior point of $a$ including no points of $c$ not on $a$. Points of the boundary of this triangle within $t$ and not on $a$ are not on $c$.

\section{§6. The approach to and crossing of a boundary.}

Definition 10. Let $P$ be an interior point of a region, $R$, and $B$ a point of the boundary $b$ of $R$. An arc of a curve, $a$, whose end points are $P$ and $B$ approaches $B$ from $P$ through $R$ if every interval of $a$, one of whose end points is $B$, contains interior points of $R$. The approach is one-sided if, besides the above condition, the arc, $a$, contains no points exterior to $R$. The approach is simple if all the points of $a$, except $B$, are interior points of $R$.

An arc $a^{\prime}$ departs from a point $B^{\prime}$ of $b$ to a point $Q$ exterior to $R$ if every interval of $a^{\prime}$ with $B^{\prime}$ as an end point contains points exterior to $R$. The departure is one-sided if, besides the above condition, the arc $a^{\prime}$ contains no points interior to $R$. The departure is simple if all the points of $a^{\prime}$ except $B^{\prime}$ are exterior to $R$.

A curve $c$ crosses the boundary in a point $B$ if, with respect to a fixed sense, $B$ is between two points $C_{1}, C_{2}$ of $c, C_{1}$ interior and $C_{2}$ exterior to $R$, in such a way that the arc $C_{1} B$ approaches $B$ through $R$ and $B C_{2}$ departs from $B$ to $C_{2}$.

A curve $c$ crosses the boundary $b$ in a pair of points $B B^{\prime}$ if, with respect to a certain sense, one are $B B^{\prime}$ of $c$ is composed entirely of boundary points and if there are two points $C_{1} C_{2}$ of $c$ such that $C_{1}$ is interior to $R$ and an are $C_{1} B$ of $c$ approaches $B$ from $C_{1}$ while $C_{2}$ is exterior to $R$ and an arc $B^{\prime} C_{2}$ departs from $B^{\prime}$ to $C_{2}$.

The crossing of a boundary is simple if both the approach and departure at the point $B$ or point pair $B B^{\prime}$ are simple.

The crossing of a straight line by a curve is a special case of the definition just given. A curve is said to cross a segment $A B$ if the curve crosses the line $A B$ in a point or a pair of points. 
TheOREM 8. Any simple curve joining an interior point of a region to an exterior point crosses the boundary in a point or a pair of points.

Proof. Let $I$ be the interior point, $O$ the exterior point, and $a$ any arc of the curve from $I$ to $O$. Let $\{A\}$ be the set of all points, $A$, of the arc $a$ such that every point following $I$ and preceding $A$ is an interior or boundary point of the region. There are such points because of condition $C$ of the definition in $\S 3$. By the ordinal continuity of $a$, the set $\{A\}$ has a first forward bound $B$, i. e., a first point in the sense from $I$ to $O$ that follows every point of $\{A\}$ except possibly $B$ itself.

The arc $B O$ of $a$ departs from $B$ to $O$ as otherwise every arc $B B^{\prime \prime}$ of $B O$ would contain only interior or boundary points of the region and thus $B$ would not be a bound of $\{A\}$. Two cases can now occur. Either $B$ is approached from $I$ by the arc $I B$ of $a$ in which case our conclusion follows, or there are points $A^{\prime}$ of $\{A\}$ such that the $\operatorname{arcs} A^{\prime} B$ include only boundary points. In the last case the set of all points, $A^{\prime}$, must have a first forward bound $B^{\prime}$ in the sense from $B$ to $I$. The point $B^{\prime}$ is evidently a boundary point and is approached from $I$ by the arc of $a, I B^{\prime}$. Thus in the second case, the boundary is crossed in the pair of points $B^{\prime} B$.

Theorem 9. If a simple closed curve crosses a side of a polygon (simple or not) in one point or point-pair, it must pass through a vertex or cross the same or another side in another point or point-pair.

Proof. Let the polygon be $\mathrm{P}_{1} \mathrm{P}_{2} \ldots \mathrm{P}_{n}$ and let the curve, $j$, cross it in a point of $\mathrm{P}_{1} \mathrm{P}_{2}$. If there is another crossing on the segment $\mathrm{P}_{1} \mathrm{P}_{2}$ or if $j$ passes through a vertex, $P_{1} \ldots P_{n}$, the theorem is verified. These cases disposed of, $\mathrm{P}_{1} \mathrm{P}_{2} \mathrm{P}_{3}$ may either be collinear or non-collinear. In the first case the original crossing may have been on $\mathrm{P}_{2} \mathrm{P}_{3}$ in which case the theorem is verified or it may have been on $\mathrm{P}_{1} \mathrm{P}_{3}$ in which case we pass to the paragraph below. In case $\mathrm{P}_{1} \mathrm{P}_{2} \mathrm{P}_{3}$ are non-collinear there must be a point $J_{1}$ of $j$ and a point $O_{1}$ common to $j$ and $\mathrm{P}_{1} \mathrm{P}_{2}$ such that in a certain sense on $j$ the arc $J_{1} O_{1}$ of $j$ approaches $O_{1}$ through the region on one side of $\mathrm{P}_{1} \mathrm{P}_{2}$; likewise there must be a point $J_{2}$ of $j$ on the opposite side of $\mathrm{P}_{1} \mathrm{P}_{2}$ from $J_{1}$ and a point $O_{2}$ common to $\mathrm{P}_{1} J_{1}$ and $j$ such that in the same sense the arc $\mathrm{O}_{2} J_{2}$ departs from $\mathrm{O}_{2}$ to $J_{2}$. Moreover the points $J_{1}$ and $J_{2}$ may be so chosen that one and only one of them lies within the triangle $\mathrm{P}_{1} \mathrm{P}_{2} \mathrm{P}_{3}$. Since $j$ crosses $\mathrm{P}_{1} \mathrm{P}_{2}$ only once, $O_{1}$ and $O_{2}$ are on the same are of $j$ with end points $J_{1} J_{2}$. The other arc, $a$, of $j$ with end points $J_{1} J_{2}$ must, by theorem 8 , cross the boundary of the triangle $\mathrm{P}_{1} \mathrm{P}_{2} \mathrm{P}_{3}$ and since it does not pass through a vertex, must either cross $\mathrm{P}_{2} \mathrm{P}_{3}$ verifying the theorem or cross $\mathrm{P}_{1} \mathrm{P}_{3}$. In the latter case, let $O_{1}^{\prime}$ be the first point in the sense from $J_{1}$ to $J_{2}$ in which $a$ meets $\mathrm{P}_{1} \mathrm{P}_{3}$ and $O_{2}^{\prime}$ the last such point. Upon the ares $J_{1} O_{1}^{\prime}$ and $O_{2}^{\prime} J_{2}$ there must be two points of $a, J_{1}^{\prime}$ and $J_{2}^{\prime}$ on opposite sides of $\mathrm{P}_{1} \mathrm{P}_{3}$ such that in opposite senses along $j$ the $\operatorname{arcs} J_{1}^{\prime} O_{1}^{\prime}$ and $J_{2}^{\prime} O_{2}^{\prime}$ approach $O_{1}^{\prime}$ and $O_{2}^{\prime}$ 
from opposite sides of $\mathrm{P}_{1} \mathrm{P}_{3}$. In case $\mathrm{P}_{1} \mathrm{P}_{3} \mathrm{P}_{4}$ are non-collinear $J_{1}^{\prime}$ and $J_{2}^{\prime}$ may be so chosen that one is interior and the other exterior to the triangle $\mathrm{P}_{1} \mathrm{P}_{3} \mathrm{P}_{4}$.

Thus whether $\mathrm{P}_{1} \mathrm{P}_{3} \mathrm{P}_{4}$ are or are not collinear we proceed as with $\mathrm{P}_{1} \mathrm{P}_{2} \mathrm{P}_{3}$, either verifying the theorem or arriving at the case $\mathrm{P}_{1} \mathrm{P}_{4} \mathrm{P}_{5}$. Continuing this process, by a finite number of steps we come to $\mathrm{P}_{1} \mathrm{P}_{n-1} \mathrm{P}_{n}$ and verify the theorem if it is not fulfilled at one of the intermediate steps.

Corollary 1. If $j_{1}$ is a simple closed curve having an arc which is a linear interval $J_{1} J_{2}$, and if the segment $J_{1} J_{2}$ is crossed by a simple closed curve $j_{2}$ in one point or point pair, then either $J_{1} J_{2}$ is crossed in another point or point pair or the non-linear arc $J_{1} J_{2}$ of $j_{1}$ has a point in common with $j_{2}$.

Proof. In case $J_{1} J_{2}$ were not crossed more than once and the other arc $J_{1} J_{2}$ of $j_{1}$ did not meet $j_{2}$, by theorem $3 J_{1}$ and $J_{2}$ could be joined by a broken line not meeting $j_{2}$ and we should thus have a contradiction with theorem 9 .

Corollary 2. Any simple closed curve $j_{1}$ having a linear arc $J_{1} J_{2}$ decomposes its plane into at least two regions.

Proof. Let $P Q$ be a linear segment crossing $J_{1} J_{2}$ in a point $O$. The region composed of all points that can be joined to $\mathrm{P}$ by broken lines not meeting $j_{1}$ is by theorem 9 separated from the region similarly connected with $Q$.

Lemma A. Any simple closed curve $j$ decomposes the plane in which it lies into at least two regions.

Proof. Let $J_{1}$ and $J_{2}$ be two points of $j$ such that the linear segment $J_{1} J_{2}$ has no point in common with $j$. Such points $J_{1} J_{2}$ exist, for if $a$ is any line joining two points of $j$, it either has an interval free of $j$ points and whose endpoints are the required points $J_{1} J_{2}$ or its points in common with $j$ constitute a single are of $j$ (theorem 6 , corollary). In the latter case any line $a^{\prime}$ joining a point of $j$ on $a$ to a point of $j$ not on $a$ evidently has the required points $J_{1} J_{2}$.

Let $t$ be a triangle about $J_{1}$ such that one of its sides meets the linear segment $J_{1} J_{2}$ in a point $O$. Let $Q^{\prime}$ and $Q^{\prime \prime}$ be two points of this side separated by $O$ and such that the linear interval $Q^{\prime} Q^{\prime \prime}$ contains no point of $j$. The existence of these points depends on the theorem that $j$ is a geometrically perfect set.

$J_{1}$ and $J_{2}$ decompose $j$ into two segments which with the linear interval $J_{1} J_{2}$ constitute two closed curves $j^{\prime}$ and $j^{\prime \prime}$. Assign the notation so that the first point, $J_{1}^{\prime}$, after $Q^{\prime \prime}$ in the sense $Q^{\prime} O Q^{\prime \prime}$ in which the boundary of $t$ meets $j$ shall be a point of $j^{\prime}$. It follows that the first point $J_{1}^{\prime \prime}$ after $Q^{\prime}$ in the sense $Q^{\prime \prime} O Q^{\prime}$ in which the boundary of $t$ meets $j$ is a point of $j^{\prime \prime}$. For if it were a point of $j^{\prime}$, the closed curve composed of the boundary of $t$ from $J_{1}^{\prime \prime}$ to $J_{1}^{\prime}$ in the sense $Q^{\prime} O Q^{\prime \prime}$ and the are common to $j$ and $j^{\prime}$ between $J_{1}^{\prime}$ and $J_{1}^{\prime \prime}$ would cross the linear segment $J_{1} J_{2}$ of $j^{\prime \prime}$ simply in $O$ and would meet $j^{\prime \prime}$ in no other point. This would contradict corollary 1 , theorem 9.

Thus $J_{1}^{\prime \prime}$ is a point of $j^{\prime \prime}$. Let $J_{0}^{\prime \prime}$ be the first point after $J_{1}^{\prime}$ in the sense $Q^{\prime} O Q^{\prime \prime}$ in which the boundary of $t$ meets $j^{\prime \prime}$. By the continuity of $j$, there 
exists a segment of the boundary of $t$ just preceding $J_{0}^{\prime \prime}$ in the sense $Q^{\prime} O Q^{\prime \prime}$ and containing no point of $j^{\prime}$ or $j^{\prime \prime}$. Let $X$ be any point of this segment. The broken line $b^{\prime \prime}$ composed of the boundary of $t$ in the sense $Q^{\prime} O Q^{\prime \prime}$ from $Q^{\prime \prime}$ to $X$ does not meet $j^{\prime \prime}$. Likewise $X$ is joined to $Q^{\prime}$ by a simple curve $c^{\prime}$ composed of the linear segment $X J_{0,1}^{\prime \prime}$, the common part of $j^{\prime \prime}$ and $j$ from $J_{0}^{\prime \prime}$ to $J_{1}^{\prime \prime *}$ and the part of the boundary of $t$ from $J_{1}^{\prime \prime}$ to $Q^{\prime}$ in the sense $Q^{\prime} O Q^{\prime \prime}$. Thus $c^{\prime}$ cannot meet $j^{\prime}$ and, applying theorem $3, c^{\prime}$ can be replaced by a broken line $b^{\prime}$ joining $X$ to $Q^{\prime}$ without meeting $j^{\prime}$. We are now ready to complete the proof of our lemma by showing that $X$ cannot be joined to $O$ by a broken line not meeting $j$.

In the sense from $X$ to $O$ any such broken line would meet the linear segments $J_{1} J_{2}$ and $Q^{\prime} Q^{\prime \prime}$ in some first point $O_{1}$. If $O_{1}$ were on $J_{1} J_{2}$, some point $S$ preceding $O_{1}$ in the sense from $X$ to $O$ could be joined to a point $B$ of $Q^{\prime} Q^{\prime \prime}$ by a segment not meeting $j^{\prime}$ or $j^{\prime \prime}$. Call $b$ the resulting broken line from $X$ to $B$. In case $O_{1}$ were not on $J_{1} J_{2}$ it would be on $Q^{\prime} Q^{\prime \prime}$ and different from $O$, and $b$ would be the broken line from $X$ to $O_{1}=B$.

If $B$ were on the same side of the line $J_{1} J_{2}$ with $Q^{\prime \prime}$ then the polygon composed of $b$ and $b^{\prime}$ and $B Q^{\prime}$ would be crossed by $j^{\prime}$ in $O$ and would meet $j^{\prime}$ in no other point, contradicting theorem 9 . If $B$ were on the opposite side of the line $J_{1} J_{2}$ from $Q^{\prime \prime}$ the polygon composed of $b$ and $b^{\prime \prime}$ and $B Q^{\prime \prime}$ would be crossed by $j^{\prime \prime}$ in $O$ and would meet $j^{\prime \prime}$ in no other point. $X$ and $O$ are therefore two points that cannot be joined by a broken line not meeting $j$.

\section{§ 7. Finite accessibility.}

Definition 11. A point $C$ of a curve $c$ is finitely accessible from a point $P$ not on $c$ if there is a broken line from $C$ to $P$ not meeting $c$ except in $C$.

Lemia $B$. If $P$ is a point not on a simple closed curve $j$, and $J_{1}$ and $J_{3}$ are any two points of $j$ finitely accessible from $P$ or limit points $\dagger$ of the points finitely accessible from $P$, then there exists a pair of points $J_{2}$ and $J_{4}$ finitely accessible from $P$ that separate $J_{1}$ and $J_{3}$.

Proof. Let $t_{1}$ be a triangle about $J_{1}$ not including $J_{3}$, and $t_{3}$ a triangle about $J_{3}$ not including any point of $t_{1}$. By condition $C$ of the definition of $j$, there is a segment of $j$ including $J_{1}$ and lying wholly within $t_{1}$; by theorem 4 there is a triangle $t_{1}^{\prime}$ about $J_{1}$ within $t_{1}$ and including no point of $j$ not on this segment. Thus every segment of $j$ with end points on $t_{1}$ which meet $t_{1}^{\prime}$ must include $J_{1}$. Similarly there is within $t_{3}$ a triangle $t_{3}^{\prime}$ such that every segment of $j$ with end points on $t_{3}$ which meets $t_{3}^{\prime}$ must include $J_{3}$.

* Of course it may happen that $J_{0}^{\prime \prime}=J_{1}^{\prime \prime}$. In this case $c^{\prime}$ is a broken line.

† On a simple closed curve the notions of ordinal and geometrical limit points are interchangable : therefore we drop the distinction. 
Let $J_{1}^{\prime}$ be a point finitely accessible from $\mathrm{P}$ within $t_{1}^{\prime}$ and $J_{3}^{\prime}$ a point finitely accessible from $\mathrm{P}$ within $t_{3}^{\prime}$. The points $J_{1}^{\prime}$ and $J_{3}^{\prime}$ are thus joined by a broken line $b$, meeting $j$ only in $J_{1}^{\prime}$ and $J_{3}^{\prime}$, which without loss of generality may be supposed simple. On this broken line let $\mathrm{P}_{1}$ be the first point in the sense from $J_{3}^{\prime}$ to $J_{1}^{\prime}$ in which it meets the boundary of $t_{1} . \quad \mathrm{P}_{1}$ lies on an interval $i_{1}$ of the boundary of $t_{1}$ containing no points of $j$ but such that its end points are points of $j$. Let $\mathrm{P}_{2}$ be the last point in the sense from $J_{3}^{\prime}$ to $J_{1}^{\prime}$ in which $b$ meets the interval $i_{1}$. In case $\mathrm{P}_{2}$ is distinct from $\mathrm{P}_{1}$ replace the portion of the broken line from $\mathrm{P}_{1}$ to $\mathrm{P}_{2}$ by the portion of $i_{1}$ from $\mathrm{P}_{1}$ to $\mathrm{P}_{2}$, calling the new broken line $b_{1}$. If $b_{1}$ crosses the interval $i_{1}, J_{2}$ and $J_{4}$ are the end points of the interval. If $b_{1}$ does not cross $i_{1}$ there must be some point $\mathrm{P}_{3}$ beyond $\mathrm{P}_{2}$ in the sense from $J_{3}^{\prime}$ to $J_{1}^{\prime}$ in which $b_{1}$ meets the boundary of $t$. The point $\mathrm{P}_{3}$ must lie on an interval $i_{3}$ of the boundary of $t_{1}$ analogous to $i_{1}$. Proceed with $i_{3}$ as with $i_{1}$. Since $J_{1}^{\prime}$ is inside $t_{1}$ and $J_{3}^{\prime}$ outside $t_{1}$ and since $b$ has but a finite number of sides, we must by repeating the process above come to a first interval $i_{l k}$, in which the boundary of $t_{1}$ is crossed by a reduced broken line $b_{k}$ from $J_{3}^{\prime}$ to $J_{1}^{\prime}$ in a point $\mathrm{P}_{k}$ or a point pair $\mathrm{P}_{k} \mathrm{P}_{k+1}$. The end points of the $j$-point free interval $i_{k}$ of the boundary of $t_{1}$ are now to be shown to be the required $J_{2}$ and $J_{4}$.

We prove first that $J_{2}$ and $J_{4}$ separate $J_{3}^{\prime}$ and $J_{1}^{\prime}$. If this were not so, let the simple clused curve formed by $b_{k}$ and the arc $J_{1}^{\prime} J_{3}^{\prime}$ of $j$ not including $J_{2}$ and $J_{4}$ be denoted by $j_{b}$. Also let $j_{i}$ denote the simple closed curve formed by $i_{k}$ and the arc $J_{2} J_{4}$ of $j$ not including $J_{3}^{\prime}$ and $J_{1}^{\prime}$. The simple closed curve $j_{b}$ would cross the are $i_{k}$ of $j_{i}$ in the point $\mathrm{P}_{\mathrm{k}}$ or point pair $\mathrm{P}_{\mathrm{k}} \mathrm{P}_{\mathrm{k}+1}$ and would meet $i_{i}$ in no other point, contrary to corollary 1 , theorem 9 .

Hence $J_{1}^{\prime}$ and $J_{3}^{\prime}$ are on different ares of $j$ with end points $J_{2}$ and $J_{4}$. But by the construction of the triangle $t_{1}^{\prime}, J_{1}$ must be on the same arc with $J_{1}^{\prime}$ and by the construction of $t_{3}^{\prime}, J_{3}$ must be on the same are with $J_{3}^{\prime}$. Hence $J_{2} J_{4}$ separate $J_{1} J_{3}$.

TheOREm 10. The set of points of a simple curve $j$, finitely accessible from a point $\mathrm{P}$ not on $j$ is everywhere dense on $j$.

Proof. Denote by $[J]$ the set of points of $j$ which are either finitely accessible from $\mathrm{P}$ or are limit points of the set of finitely accessible points. The theorem amounts to showing that $\left[J^{\prime}\right]$ is identical with $j$. But if any point $J_{0}$ of $j$ should not belong to [ $\left.J^{\prime}\right]$ it would lie on an arc of $j$ free of points $J^{\prime}$ and having two points of $\left[J^{\prime}\right]$ as end points. This would contradict lemma $B$. 


\section{§ 8. Decomposition of a plane by a simple closed curve.}

Lemma $C$. Any simple closed curve of which one are is a linear interval decomposes its plane into two open regions.

Proof. It has been shown (corollary 2, theorem 9) that $j$ decomposes the plane into at least two regions. The regions are open because a supposed frontier point of the set of points $[\mathrm{P}]$ that can be joined to a point $\mathrm{P}_{0}$ not on $j$ could, if not itself a point of $j$, be surrounded by a triangle not meeting $j$ and containing points of $[\mathrm{P}]$; it would therefore be an interior point of $[\mathrm{P}]$, contrary to hypothesis.

By theorem 10 every point $O$ of the straight are of $j$ is finitely accessible from any point of the plane. Thus if there were three distinct regions there would be three segments meeting in $O$ and one lying in each of the three regions. But as two of these must lie on the same side of the straight segment of $j$ they could be joined by a straight segment not meeting $j$, contrary to the hypothesis that the three regions are separated from one another by $j$. Hence $j$ decomposes the plane into two and only two open regions.

Theorem 11. Every simple closed curve, $j$, decomposes its plane into two open regions.

Proof. By lemma $A$ the curve decomposes the plane into at least two regions which by the reasoning of the first paragraph of the proof of lemma $C$ are open regions. Let $\mathrm{P}$ be any point not on $j$ and let $\mathrm{P} J_{1}$ and $\mathrm{P} J_{2}$ be two linear intervals meeting $j$ only in $J_{1}$ and $J_{2} . \quad J_{1}$ and $J_{2}$ exist because $J$ is a perfect set of points. Let $Q$ be any point not on $j$ and not in the same region with $\mathrm{P}$ and let $J_{3}$ be a point on $j$ such that the linear segment $Q J_{3}$ does not meet $j$ and such that $J_{3}$ is distinct from $J_{1}$ and $J_{2}$. Then $Q J_{3}$ does not meet $\mathrm{P} J_{1}$ or $\mathrm{P} J_{2}$ and $Q$ can by theorem 10 be joined by a broken line not meeting $\mathrm{P} J_{1}, \mathrm{P} J_{2}, Q J_{3}$, or $j$ except in $J_{4}$ to a point $J_{4}$ of $j$ in the order $J_{1} J_{2} J_{3} J_{4}$. The broken line $J_{1} \mathrm{P} J_{2}$, the points between $J_{2}$ and $J_{3}$ in the sense $J_{1} J_{2} J_{3}$, the broken line $J_{3} Q J_{4}$ and the points between $J_{4}$ and $J_{1}$ in the sense $J_{1} J_{2} J_{3}$ constitute a simple closed curve $j^{\prime}$ of the type which we have proved to decompose the plane into two and only two regions. The points of the segments $J_{1} J_{2}$ and $J_{3} J_{4}$ in the sense $J_{1} J_{2} J_{3}$, are not points of $j^{\prime}$ and must lie both in the same region or in opposite regions with respect to $j^{\prime}$. If they were in the same region a point in the region not containing the segments $J_{1} J_{2}$ and $J_{3} J_{4}$ could by theorem 10 be joined by broken lines not meeting $j$ to $\mathrm{P}$ and $Q$, thus contradicting the hypothesis that $\mathrm{P}$ and $Q$ are in different regions.

Having shown that the ares $J_{1} J_{2}$ and $J_{3} J_{4}$ (in the fixed sense $J_{1} J_{2} J_{3}$ ) are in opposite regions with respect to $j^{\prime}$ we are ready to complete the proof that $j$ does not decompose the plane into more than two regions. A point $R$ in a sup- 
posed third region could be joined because of theorem 10 by a broken line not meeting $j$ except in its end-point to a point $J_{5}$ of $J_{1} J_{2}$ and by a similar broken line to a point $J_{6}$ of $J_{3} J_{4}$. Since $R$ would not be in the same region with $\mathrm{P}$ or $Q$ these broken lines would not meet the broken line part of $j^{\prime}$. Thus we should have two points $J_{5}$ and $J_{6}$ in opposite regions with respect to $j^{\prime}$ joined by a broken line not meeting $j^{\prime}$ contrary to lemma $C$. Hence $j$ decomposes the plane into not more than two, and therefore into exactly two, open regions. 